\title{
Proposal of a Mixed-Methods Study to Develop and Validate the Malaysian Anti-Hypertensive Agents Compliance Scale in Hypertensive Patients
}

Sheng Qian Yew ( $\nabla$ ysq_sam@yahoo.com )

Universiti Putra Malaysia https://orcid.org/0000-0002-2430-1540

Ahmad lqmer Nashriq Bin Mohd Nazan

Universiti Putra Malaysia

Kit Aun Tan

Universiti Putra Malaysia

Anisah Baharom

Universiti Putra Malaysia

Study protocol

Keywords: Hypertension, drug compliance, mixed methods

Posted Date: November 4th, 2020

DOl: https://doi.org/10.21203/rs.3.rs-100174/v1

License: (c) (i) This work is licensed under a Creative Commons Attribution 4.0 International License.

Read Full License 


\section{Abstract}

Background: There is still insufficient blood pressure control in hypertensive patients although antihypertensive medications are highly available. Determinants of compliance and non-compliance with such medications in hypertensive patients have not been well documented. In response, the objective of the present study is twofold: to identify themes of compliance and non-compliance towards antihypertensive medications in hypertensive patients (Study 1) and to develop and validate the Malaysian Anti-Hypertensive Agents Compliance Scale (MAACS) (Study 2).

Methods: This study has an exploratory mixed-methods design and will be conducted from December 2020 to December 2021. In Study 1, we will recruit hypertensive patients from two selected clinics to participate in a semi-structured interview session. Recruitment of participants will be terminated once thematic saturation is achieved. Coding and thematic analyses will be performed to identify the themes. In Study 2, based on the themes generated from Study 1, we will develop a Malaysian version of the AntiHypertensive Agents Compliance Scale (MAACS). Four hundred hypertensive patients will be randomly selected. Of these, data from 200 participants (serving as the calibration sample) will be subjected to exploratory factor analysis and data from 200 participants (serving as the validation sample) will be subjected to confirmatory factor analysis. Factor structure, concurrent validity, and reliability of the MAACS will be statistically tested.

Discussion: With the presence of the MAACS, public health officials and health care providers can gather crucial information regarding barriers and facilitators to hypertensive treatment compliance and design effective health promotion programme in an attempt to reduce complications of uncontrolled hypertension.

\section{Background}

Hypertension is an increasingly important public health issue with a global prevalence of $31.1 \%$ [1]. The prevalence of hypertension was $35.3 \%$ in Malaysian adults aged 18 years and above [2]. Moreover, the number of adults with hypertension in 2025 is predicted to increase by about a total of 1.56 billion globally [3]. Such increase is owing to the issues of ageing as well unhealthy and sedentary lifestyles [4]. There is still insufficient blood pressure control in hypertensive patients although anti-hypertensive drugs are highly available $[5,6]$. In developed countries, the compliance with medication among patients with chronic diseases averages only around 50\% [7]. This situation was reported to be even worse for developing countries due to poor accessibility to medications and health care services [7].

In Malaysia, the data on medication compliance are scarce. Till date, only a small number of medication compliance studies were available in Malaysia. In one study, Ramli et al. reported that only $53.4 \%$ of patients had complied to the prescribed hypertensive medications and the control of hypertension was far beyond satisfactory [8]. In another study, Turki and Sulaiman found that only half of the hypertensive patients had complied to their prescribed hypertensive medications [9]. These statistics are not only 
alarming but should serve as a wake-up call for research into non-compliance with anti-hypertensive drugs.

The determinants of compliance and non-compliance in relation to hypertensive treatment warrant scientific investigations. Many researchers have attempted to develop scales for measuring compliance towards anti-hypertensive treatments. These scales include but are not limited to the nine-item Brief Medication Questionnaire (BMQ) [10], the four-Item Morisky Medication Adherence Scale (MMAS-4) [11], the eight-Item Morisky Medication Adherence Scale (MMAS-8) [12], the 25-item Maastricht Utrecht Adherence in Hypertension (MUAH-25) [13], the 16-item Maastricht Utrecht Adherence in Hypertension (MUAH-16) [14], the 28-item Treatment Adherence Questionnaire for Patients with Hypertension (TAQPH) [15], the 14-item Hill-Bone Compliance Scale (HBSC) [16], and the seven-item Self-Efficacy for Appropriate Medication Use Scale (SEAMS) [17]. Of note, the BMQ, MMAS-4, MMAS-89, HBSC, and SEAMS were developed from quantitative approach, whereas the MUAH-16 and the MUAH-25 were developed from qualitative approach. Only the TAQPH was developed from mixed method approach. As far as theoretical framework is concerned, only the SEAMS can trace its roots back to the Bandura's Social Cognitive Theory [17]. The use of conceptual framework in the development of the remaining scales was unclear.

\section{Hypertensive Treatment Compliance as a Multidimensional Construct}

Drug compliance is a multidimensional construct encompassing patient- (e.g., the MUAH-16, MUAH-25 \& the TAQPH), health system- (e.g., the MUAH-16 \& the MUAH-25), therapy- (e.g., the MUAH-16, the MUAH25, \& the TAQPH), and condition-related factors (e.g., the SEAMS) [18]. In particular, the MUAH-16 [14], the MUAH-25 [13], the TAQPH [15], and the SEAMS [17] are multidimensional, whereas the MMAS-4 [11], the MMAS-8 [12], and the HBCS [16] are unidimensional. Often, patients are simply classified as compliant or non-compliant to a drug therapy. However, if the determinants for their compliance and non-compliance were better understood via a multi-dimensional scale, intervention programs to improve drug compliance could be more optimised, and thus more successful. Utilising a multidimensional scale may prevent considerable loss of information as seen in unidimensional scale [19].

\section{Hypertensive Treatment Compliance as a Culturally Specific Construct}

Health care utilisation can vary across different countries and cultures [20]. The self-reported MMAS-8 has been widely used to assess the drug compliance in hypertensive patients, thanks to its broad validation and translation studies and outstanding validity and reliability properties [21]. Other hypertension-related scales have not been validated outside the setting which they were originally developed. One such example is the HBCS. The scale was developed and validated in an American African community [22]. It appears that the HBCS has a higher performance for African than non-African populations [23]. This scale has been suggested as suitable for use in studies specific for hypertension in a predominantly African population. 


\section{Hypertensive Treatment Compliance as a Reliable and Valid Construct}

Psychometric properties of a scale can be assessed through reliability and validity. It has been suggested that a Cronbach's alpha of at least .70 is required to indicate adequate internal consistency [24]. With such standard, it seems that the MMAS-4 [11] and the MUAH-16 [14] fail to demonstrate adequate internal consistency. Some scales have demonstrated satisfactory reliability in the original scale development studies, but they did not perform well psychometrically when used in patients residing in different countries. The MMAS-8 was translated to French [25] and Urdu [26] versions. The Cronbach's alphas were .83 for the original version, .54 for the French version, and .71 for the Urdu version. When taking validity of the scales into account, only the HBCS [16] and TAQPH [15] reported evidence for content validity. Only the MUAH-16 [14] and the MUAH-25 [13] have documented evidence for convergent validity, whereas only the MMAS-4, the MMAS-8, the TAQPH, and the SEAMS have documented evidence for criterion-related validity. No construct validity information is yet available for the BMQ [10].

\section{The Present Study}

No single measure that can be appropriately administered in the context of Malaysian hypertensive patients is available. Some existing scales are unidimensional (e.g., the MMAS-4, the MMAS-8, \& the HBCS) and have low reliability and validity estimates (e.g., the MMAS-4 \& the MUAH-16). Other scales are not built upon a solid conceptual framework (e.g., the BMQ, the MMAS-4, the MMAS-8, the MUAH-16, the MUAH-25, the HBCS, \& the TAQPH) and are culturally specific (e.g., the HBCS). There exists a timely need to develop a theoretically driven, multidimensional, reliable, and valid scale for use in assessing compliance and non-compliance towards anti-hypertensive medications in Malaysia. Hence, the objective of the present study is to identify themes of compliance and non-compliance towards anti-hypertensive medications in hypertensive patients (Study 1) and to develop and validate the Malaysian AntiHypertensive Agents Compliance Scale (MAACS) (Study 2). This study has an exploratory mixedmethods design and will be conducted from December 2020 to December 2021.

\section{Methods}

\section{STUDY 1}

\section{Objectives}

In Study 1, we will conduct a qualitative study to identify themes of compliance and non-compliance towards anti-hypertensive drugs in hypertensive patients. An approach of phenomenology will be utilised to study the phenomenon of anti-hypertensive treatment compliance among hypertensive patients.

\section{Methods}

\section{Participants}


For Study 1, data collection and participant recruitment will take place at health clinics located in Kuala Lumpur. In Kuala Lumpur, there are 13 health clinics. A two-stage sampling method will be employed to recruit study participants. Only two health clinics will be randomly selected. A purposeful sampling will be conducted to select eligible hypertensive patients attending the participating health clinics from December 2019 to December 2020. We will identify the hypertensive patients from the patients' registry. Potential participants will be reached through phone call, subject to the inclusion and exclusion criteria listed below. The interview session will include 10 to 20 patient participants. These participants will not be recruited for Study 2 to avoid confounding bias.

\section{Participants Recruitment}

On inclusion criteria, participants will be recruited for this study if they are (a) at least 18 years old, (b) diagnosed with hypertension defined as persistent elevation of systolic blood pressure of $130 \mathrm{mmHg}$ or greater and/or diastolic blood pressure of $80 \mathrm{mmHg}$ or greater, or currently prescribed with antihypertensive drugs, (c) have received anti-hypertensive drug treatment for at least 6 months, and (d) able to communicate in English language or Malay language. Psychiatric patients are eligible, if they are (a) aged at least 18 years old, (b) able to give consent independently, and (c) have no suicidal tendency at the time of study recruitment. Written consent forms, patient information sheets describing the study, and the ethical approval letters will be provided to the participants who agree to participate to the study.

On exclusion criteria, no participants will be recruited for this study if they are diagnosed with (a) secondary hypertension such as parenchymal kidney disease, renovascular disease, primary aldosteronism, Cushing syndrome, and phaeochromocytoma, (b) malignancies, (c) cognitive impairment and mental health disorders such as schizophrenia, major depressive disorder, generalised anxiety disorder, and dementia, and (d) pregnant. Patient history and medical record will be used to establish exclusion criteria.

\section{Data Collection}

A semi-structured interview schedule will be developed with reference to the Capability, Opportunity, Motivation, and Behaviour (COM-B) model so as to explore the experience of taking anti-hypertensive drugs, including the determinants of them being non-compliant towards the prescribe medications (Appendix A). The COM-B model consists of 3 main domains namely capability, opportunity, and motivation. The interplay of capability, opportunity, and motivation could affect individuals' behaviour of taking medication [27]. In particular, we will use the four-step Interview Protocol Refinement (IPR) Framework to develop the interview schedule to ensure validity and reliability [28]. In Step 1, we will design a series of open-ended questions in the interview schedule in line with our research objective as described above. In Step 2, we will construct all interview schedule questions in an inquiry-based conversation fashion (e.g., can you please share with me regarding your experience with high blood pressure?) and in English so that participants from different sociodemographic subgroups can easily comprehend the questions. In Step 3, we will invite two members of the supervisory committee and an external primary care physician to independently review the interview schedule and provide feedback. In 
Step 4, we will test the newly developed interview schedule with two hypertensive patients, one from each health clinic. Subsequently, an independent translator will translate the validated interview schedule into Malay and Mandarin.

All interview sessions will be conducted in a quiet meeting room at the clinics. We will begin our interviews by discussing the purposes of the interview. In an effort to build rapport, participants will be asked to introduce themselves. They will also be encouraged to freely share their own thoughts and ideas. We will elicit the themes of compliance and non-compliance towards anti-hypertensive drugs among the participants through all questions in the interview schedule. We aim to complete the interviews within 45 minutes. All interviews will end with the interviewer's reflection. All interviews will be audiotaped and transcribed verbatim. Recruitment of participants will proceed until the point of thematic saturation. All data collected will remain private and confidential.

\section{Data Analysis}

After each interview, we will save the audio files in a password-secured laptop and transcribe verbatim the audio using NVIVO software. Transcripts will be then checked against the audio recording of each interview to ensure accuracy. Participant names will be replaced with coded names for confidentiality purpose. Should the interviews be conducted in Malay or Mandarin, we will translate the recordings back into English. A constant comparative method will be used for analysis. Specifically, we will read through the transcripts before extracting and coding the meaning units. Then, we will categorize the codes. We will ensure the quality and rigor of the qualitative inquiry through the tests of credibility, transferability, dependability, and confirmability.

We aim to achieve the credibility of the current study through triangulation and member checks. In the process of triangulation, two independent investigators will analyse the same set of data independently and to compare findings. Member checks will also be performed by taking the analysed data back to the hypertensive patients for verification. We will ensure dependability through peer review and researcher reflexivity. Peer review will be done by inviting a qualitative researcher to go through part of the raw data and to examine whether the research findings are plausible in accordance to the interview data. In addition, the interviewers will also clearly mention their dispositions, biases, worldview, assumptions, theoretical orientation, and relationship to the study that may affect the investigation. We aim to achieve transferability through rich and thick description of the study setting and the characteristics of the participants (e.g., age, gender, ethnicity, \& level of education of the hypertensive patients). Confirmability will be ensured through the provision of verbatim [29].

All audio recordings will be de-identified, and there will be no mention of personal identifying information (e.g., names, identification card number, and address) during the interview. All audio recordings are for transcription purposes and will not be copied or transferred to any other parties or used for any other purposes. After transcription, the audio recordings will be disposed securely. We will store all audio recordings in the form of voice recording MP3 until the completion of transcription, while the transcription 
will be saved as Microsoft Word format in a password-secured computer. Only the principle investigator and coordinator investigator will have access to the data.

\section{STUDY 2 \\ Objective}

We will conduct a quantitative study to develop and validate the Malaysian Anti-hypertensive Agents Compliance Scale (MAACS) in a sample of hypertensive patients. This quantitative study is crosssectional by design.

\section{Methods}

\section{Item Generation}

The preliminary version of the MAACS will consist of themes that generated from Study 1 , which aimed at identifying determinants of hypertension treatment compliance. Based on these themes, we will create the preliminary MAACS items. Thirty participants will be asked to individually and independently evaluate and score each preliminary MAACS item for its appropriateness, representativeness, and explicitness using a 4-point Likert scale ranging from 1 (irrelevant and should be deleted) to 4 (relevant, clear, and precise). Statistically, only items with a mean score of 3.0 or above will be retained. The decision about whether to retain any preliminary MAACS items scoring below 3.0. rests with the experts. An experts' panel meeting will be conducted by A.I.N.M.N., K.A.T., S.Q.Y, and A.B. for determining the appropriateness of either deleting or retaining each item. Hence, the preliminary version of the MAACS.

\section{The Pilot Version}

Pilot testing helps to ensure that items are meaningful to the Malaysian hypertensive population. The pilot study is needed to minimise misunderstanding and subsequent measurement error [30], and to eliminates poorly worded items [31]. We will conduct cognitive interviews with 5 to 15 hypertensive patients. During the interview, participants will have chance to augment, clarify, and modify each preliminary MAACS item so that the item is clear, culturally accepted, and not time consuming. This process will result in the pilot version of the MAACS.

\section{Participants Recruitment}

In Study 2, data collection and participant recruitment will also take place at the two participating health clinics located in Kuala Lumpur as in Study 1. A random sampling will be conducted to select eligible hypertensive patients who attend these two clinics from December 2019 to December 2020.

As for sample size calculation, we would require at least 200 participants when taking structural equation modelling into consideration [32]. Hence, a final sample of 400 participants will be recruited. Of these, data from 200 participants (serving as the calibration sample) will be subjected to exploratory factor 
analysis (EFA) and data from 200 participants (serving as the validation sample) will be subjected to confirmatory factor analysis (CFA). All participants will be selected according to the same inclusion and exclusion criteria as described in Study 1.

\section{Measures}

Participants will complete a research questionnaire containing sociodemographic items (e.g., age, gender, ethnicity, level of education, marital status, employment status, duration of hypertension, blood pressure, \& medication use), the pilot version of the MAACS, and the TAQPH.

\section{Data Analytic Plan}

\section{The Modified Version}

We will examine the factor structure of the pilot version of the MAACS with the calibration sample. A principal-component analysis with promax rotation will be conducted to determine the factor structure of the pilot version of the MAACS. In particular, the following criteria will be used to determine the number of factors in the instrument: eigenvalues $>1$, and items with loadings of 4 or greater on any one factor. When this was done, we will obtain the modified version of the MACCS.

\section{The Final Version}

We will examine the factor structure of the modified version of the MACCS with the validation sample. CFA will be performed to examine the goodness-of-fit of the MACCS measurement model. In particular, The MACCS measurement model will be evaluated by chi-square $\left(\chi^{2}\right)$ test, Goodness of Fit Index (GFI), Adjusted Goodness of Fit Index (AGFI), Comparative Fit Index (CFI), Root Mean Squared Error of Approximation (RMSEA), Non-Normed Fit Index (NNFI) and Standard Root Meansquare Residual (SRMR). Acceptable model fit is indicated by $\chi^{2} / d f<3.0$, RMSEA $<.08$ and SRMR $<.8$. The values of GFI, AGFI, CFI, and NNFI indices should be .90 or greater [33]. This will result in the final version of the MAACS.

\section{Test for Validity}

To establish concurrent validity, we will examine the Pearson's $r$ correlations between the final version of the MAACS and the TAQPH [15]. The 28-item TAQPH is a self-report scale designed to identify determinants of compliance and non-compliance towards anti-hypertensive medications [15]. Participants rate items on a 4-point Likert scale ranging from 1 (never) to 4 (all of the time). The TAQPH has 2 domains: medication and lifestyle. Scores on these domains are summed to obtain a total score with higher scores representing better compliance.

\section{Test for Reliability}

The internal consistency of the final version of the MAACS will be measured by Cronbach's alpha coefficient. An alpha coefficient $>.80$ is referred to as high [34].

\section{Discussion}


The objective of the present study is twofold. We aimed to identify, through quantitative inquiries, the determinants of compliance and non-compliance towards anti-hypertensive medications among the hypertensive patients. We also aimed to develop and validate an anti-hypertensive drug compliance scale, named as the Malaysia Anti-hypertensive Agents Compliance Scale (MAACS), through quantitative approach. With the presence of the MAACS, public health officials and health care providers can gather crucial information regarding barriers and facilitators to hypertensive treatment compliance and design effective health promotion programme in an attempt to reduce complications of uncontrolled hypertension.

\section{Declarations}

\section{Ethics approval and consents to participate:}

Ethical approval is granted by the Medical Research Ethics Committee, Ministry of Health, Malaysia (MREC) prior to the start of the study (reference no: NMRR-18-3251-44694). All researchers involved in this study will adhere to the principles of the Declaration of Helsinki and the Malaysian Good Clinical Practice Guidelines. We will seek informed consents from all participants prior to their participation to the current study.

\section{Consent for publication:}

All research results should only be published with the consent of the principle investigator. No personal information of subjects will be published. Permission from the Head of Department of Department of Community Health, Universiti Putra Malaysia as well as the Director General of Health, Malaysia will be obtained prior to publication. All participants will be completely anonymised.

\section{Availability of data and materials:}

The datasets that will be collected during the current study are available from the corresponding author on reasonable request.

\section{Competing interests:}

The authors declare that they have no competing interests.

\section{Funding:}

This research received no external funding. 


\section{Authors' contributions:}

A.I.N.M.N., K.A.T, S.Q.Y, and A.B are involved in the conceptualisation of the current study. A.I.N.M.N. and K.A.T. are involved in the design of the methodology. S.Q.Y. prepared the original draft. K.A.T is responsible in the review and editing of the manuscript.

\section{Acknowledgements:}

The authors would like to acknowledge Dr. Wong Ping Foo (the director of Cheras Baru Health Clinic) and Dr. Baizury Binti Bashah (the director of Kuala Lumpur Health Clinic) for their permission to conduct the present study at the respective clinics.

\section{References}

1. Mills KT, Stefanescu A, He J. The global epidemiology of hypertension. Nature Reviews Nephrology. 2020;16(4):223-37.

2. Malaysia MoH. Clinical Practice Guidelines: Management of Hypertension. In: Malaysia AoM, editor. 5 ed. Putrajaya, Malaysia2018.

3. Kearney PM, Whelton M, Reynolds K, Muntner P, Whelton PK, He J. Global burden of hypertension: analysis of worldwide data. Lancet (London, England). 2005;365(9455):217-23.

4. Mills KT, Bundy JD, Kelly TN, Reed JE, Kearney PM, Reynolds K, et al. Global Disparities of Hypertension Prevalence and Control: A Systematic Analysis of Population-Based Studies From 90 Countries. Circulation. 2016;134(6):441-50.

5. Annamalai C, Govindaraja C, Chandramouli C. Prevalence, awareness and control of hypertension in estate workers in Malaysia. North American Journal of Medical Sciences. 2011;3(12):540-3.

6. Gee ME, Bienek A, McAlister FA, Robitaille C, Joffres M, Tremblay MS, et al. Factors associated with lack of awareness and uncontrolled high blood pressure among Canadian adults with hypertension. The Canadian Journal of Cardiology. 2012;28(3):375-82.

7. Adherence to long-term therapies: evidence for action. Sabaté E, World Health O, editors. Geneva: World Health Organization; 2003.

8. Ramli A, Ahmad NS, Paraidathathu T. Medication adherence among hypertensive patients of primary health clinics in Malaysia. Patient preference and adherence. 2012;6:613-22.

9. Turki AK, Sulaiman SAS. Elevated blood pressure among patients with hypertension in general hospital of Penang, malaysia: dose poor adherence matter? :9.

10. Svarstad BL, Chewning BA, Sleath BL, Claesson C. The Brief Medication Questionnaire: a tool for screening patient adherence and barriers to adherence. Patient Education and Counseling. 1999;37(2):113-24. 
11. Morisky DE, Green LW, Levine DM. Concurrent and predictive validity of a self-reported measure of medication adherence. Medical Care. 1986;24(1):67-74.

12. Morisky DE, Ang A, Krousel-Wood M, Ward HJ. Predictive Validity of A Medication Adherence Measure in an Outpatient Setting. Journal of clinical hypertension (Greenwich, Conn). 2008;10(5):348-54.

13. Wetzels G, Nelemans P, van Wijk B, Broers N, Schouten J, Prins M. Determinants of poor adherence in hypertensive patients: development and validation of the "Maastricht Utrecht Adherence in Hypertension (MUAH)-questionnaire". Patient Education and Counseling. 2006;64(1-3):151-8.

14. Cabral AC, Castel-Branco M, Caramona M, Fernandez-Llimos F, Figueiredo IV. Developing an adherence in hypertension questionnaire short version: MUAH-16. Journal of Clinical Hypertension (Greenwich, Conn). 2018;20(1):118-24.

15. Ma C, Chen S, You L, Luo Z, Xing C. Development and psychometric evaluation of the Treatment Adherence Questionnaire for Patients with Hypertension. Journal of Advanced Nursing. 2012;68(6):1402-13.

16. Kim MT, Hill MN, Bone LR, Levine DM. Development and testing of the Hill-Bone Compliance to High Blood Pressure Therapy Scale. Progress in Cardiovascular Nursing. 2000;15(3):90-6.

17. Risser J, Jacobson TA, Kripalani S. Development and psychometric evaluation of the Self-efficacy for Appropriate Medication Use Scale (SEAMS) in low-literacy patients with chronic disease. Journal of Nursing Measurement. 2007;15(3):203-19.

18. Jin J, Sklar GE, Min Sen Oh V, Chuen Li S. Factors affecting therapeutic compliance: A review from the patient's perspective. Therapeutics and Clinical Risk Management. 2008;4(1):269-86.

19. Brenninkmeijer V, VanYperen N. How to conduct research on burnout: advantages and disadvantages of a unidimensional approach in burnout research. Occupational and Environmental Medicine. 2003;60(Suppl 1):i16-i20.

20. González-Calvo J, González VM, Lorig K. Cultural diversity issues in the development of valid and reliable measures of health status. Arthritis Care and Research: The Official Journal of the Arthritis Health Professions Association. 1997;10(6):448-56.

21. De las Cuevas C, Peñate W. Psychometric properties of the eight-item Morisky Medication Adherence Scale (MMAS-8) in a psychiatric outpatient setting. International Journal of Clinical and Health Psychology : IJCHP. 2015;15(2):121-9.

22. Lavsa SM, Holzworth A, Ansani NT. Selection of a validated scale for measuring medication adherence. Journal of the American Pharmacists Association: JAPhA. 2011;51(1):90-4.

23. Krousel-Wood M, Joyce C, Holt EW, Levitan EB, Dornelles A, Webber LS, et al. Development and evaluation of a self-report tool to predict low pharmacy refill adherence in elderly patients with uncontrolled hypertension. Pharmacotherapy. 2013;33(8):798-811.

24. Taber K. The Use of Cronbach's Alpha When Developing and Reporting Research Instruments in Science Education. 2017. 
25. Korb-Savoldelli V, Gillaizeau F, Pouchot J, Lenain E, Postel-Vinay N, Plouin P-F, et al. Validation of a French version of the 8-item Morisky medication adherence scale in hypertensive adults. Journal of Clinical Hypertension (Greenwich, Conn). 2012;14(7):429-34.

26. Saleem F, Hassali MA, Akmal S, Morisky DE, Atif M, Al-Qazaz HK, et al. Translation and validation study of Morisky Medication Adherence Scale (MMAS): the Urdu version for facilitating personcentered healthcare in Pakistan. International Journal of Person Centered Medicine. 2012;2(3):38490.

27. Michie S, van Stralen MM, West R. The behaviour change wheel: A new method for characterising and designing behaviour change interventions. Implementation Science : IS. 2011;6:42.

28. Castillo-Montoya M. Preparing for Interview Research: The Interview Protocol Refinement Framework. The Qualitative Report. 2016;21(5):811-31.

29. Merriam SB. Qualitative Research: A Guide to Design and Implementation. 3 edition ed. San Francisco: Jossey-Bass; 2009.

30. Boateng GO, Neilands TB, Frongillo EA, Melgar-Quiñonez HR, Young SL. Best Practices for Developing and Validating Scales for Health, Social, and Behavioral Research: A Primer. Frontiers in Public Health. 2018;6.

31. Beatty PC, Willis GB. Research Synthesis: The Practice of Cognitive Interviewing. Public Opinion Quarterly. 2007;71(2):287-311.

32. Kline RB. Principles and Practice of Structural Equation Modeling, Third Edition. 3rd edition ed. New York: The Guilford Press; 2010.

33. El-Sheikh AA, Abonazel MR, Gamil N. A Review of Software Packages for Structural Equation Modeling: A Comparative Study. Applied Mathematics and Physics. 2017;5(3):85-94.

34. Taber KS. The Use of Cronbach's Alpha When Developing and Reporting Research Instruments in Science Education. Research in Science Education. 2018;48(6):1273-96.

\section{Supplementary Files}

This is a list of supplementary files associated with this preprint. Click to download.

- SupplementaryMaterial.docx 